\title{
A time-dependent search for high-energy neutrinos from bright GRBs with ANTARES
}

\author{
Silvia Celli ${ }^{1,2,3, a}$ \\ On behalf of the ANTARES Collaboration \\ ${ }^{1}$ Gran Sasso Science Institute, Viale Francesco Crispi 7, 67100 L'Aquila, Italy \\ ${ }^{2}$ INFN-Sezione di Roma, Piazzale Aldo Moro 2, 00185 Roma, Italy \\ ${ }^{3}$ Dipartimento di Fisica dell'Università La Sapienza, Piazzale Aldo Moro 2, 00185 Roma, Italy
}

\begin{abstract}
Astrophysical point-like neutrino sources, like Gamma-Ray Bursts (GRBs), are one of the main targets for neutrino telescopes, since they are among the best candidates for Ultra-High-Energy Cosmic Ray (UHECR) acceleration. From the interaction between the accelerated protons and the intense radiation fields of the source jet, charged mesons are produced, which then decay into neutrinos. The methods and the results of a search for high-energy neutrinos in spatial and temporal correlation with the detected gamma-ray emission are presented for four bright GRBs observed between 2008 and 2013: a time-dependent analysis, optimised for each flare of the selected bursts, is performed to predict detailed neutrino spectra. The internal shock scenario of the fireball model is investigated, relying on the neutrino spectra computed through the numerical code NeuCosmA. The analysis is optimized on a per burst basis, through the maximization of the signal discovery probability. Since no events in ANTARES data passed the optimised cuts, $90 \%$ C.L. upper limits are derived on the expected neutrino fluences.
\end{abstract}

\section{Introduction}

The search for neutrinos from astrophysical sources is motivated by the still poor understanding of the origin of UHECRs. GRBs are huge explosive phenomena, that offer a promising environment for proton and heavier nuclei acceleration at shock fronts. Photo-hadronic interactions produce highenergy gamma-rays and neutrinos mainly through the following resonant channel:

$$
p+\gamma \stackrel{\Delta^{+}}{\longrightarrow}\left\{\begin{array}{ll}
p+\pi^{0} \\
n+\pi^{+}
\end{array} \quad \begin{array}{l}
\pi^{0} \longrightarrow \gamma+\gamma \\
n \longrightarrow p+e^{-}+\bar{v}_{e} \\
\pi^{+} \longrightarrow \mu^{+}+v_{\mu} \\
\mu^{+} \longrightarrow e^{+}+v_{e}+\bar{v}_{\mu}
\end{array}\right.
$$

In addition, kaon production leads to a high-energy component in neutrino spectra. The search for such high-energy neutrinos from bright sources is motivated by the fact that theoretical GRB-models, as the internal shock scenario of the fireball model [1], predict a neutrino flux linearly scaled with the detected $\gamma$-ray flux. Hence, after a brief description of the ANTARES detector in Sec. 2, the selection

\footnotetext{
a e-mail: silvia.celli@gssi.infn.it
} 
of bright sources is introduced in Sec. 3. The methods of the time-dependent analysis are discussed in Sec. 4. Finally, in Sec. 5, results are presented and conclusions are derived.

\section{The ANTARES neutrino telescope}

The ANTARES detector [2] is the largest operational neutrino telescope of the Northern hemisphere, located in the Mediterranean sea, at a depth of about $2475 \mathrm{~m}$ : it is composed by 12 vertical strings, instrumented with 885 photo-multipliers, detecting the Cherenkov light induced by the passage in water of ultra-relativistic charged particles. The search for astrophysical point-like neutrino sources is among the ANTARES scientific goals: the signature of a point source is a cluster of events in the defined direction of the sky, where the source is located. In order to reduce the huge background of atmospheric muons, the search is performed by selecting up-going track-like events: GRBs from below ANTARES horizon are therefore considered in this search for muon neutrinos. Moreover, since GRBs are transient sources, a significant suppression of the background is further achieved by considering only the reduced time window corresponding to the bursting event. This constitutes the main difference with respect to steady source searches.

\section{Bright GRB selection and time-dependent neutrino spectra}

Bright GRBs are here investigated since, according to the fireball model, neutrino fluxes are expected to scale with gamma-ray fluxes: GRBs between 2008 and 2013 are selected from the Fermi and Swift online catalogues and from the Konus-Wind GCNs with $\gamma$-ray fluence $\mathrm{F}_{\gamma}>10^{-4} \mathrm{erg} / \mathrm{cm}^{2}$; it is also required that the sources have measured redshift and are below the ANTARES horizon at the trigger time (while ANTARES is taking physics data). Four GRBs satisfied such selection criteria: GRB 080916C, GRB 110918A, GRB 130427A and GRB 130505A. Their $\gamma$-ray light curves are represented in Fig. 2(a). In order to compute the neutrino spectra of each GRB according to the internal shock fireball model, the parameters measured by the $\gamma$-ray satellites are used: the detailed computation of neutrino emissions refers to single bursting episodes present in each GRB light curve. Such parameters are presented in Tab. 1. In this way, a time-dependent neutrino spectrum is predicted: the total expected fluence per burst is then obtained summing up the contributions from each time bin. The resulting neutrino expectation for GRB 080916C is presented in Fig. 2(b). The simulation through the numerical NeuCosmA [7] assumes $\Gamma=316$ for the bulk Lorentz factor, $\mathrm{f}_{p}=10$ for the baryonic content, $\epsilon_{e}=\epsilon_{B}=0.1$ as fraction of the jet kinetic energy transferred to electrons and to magnetic field, $\left\langle x_{p \rightarrow \pi}\right\rangle=0.2$ as average fraction of energy transferred from proton to pion. 

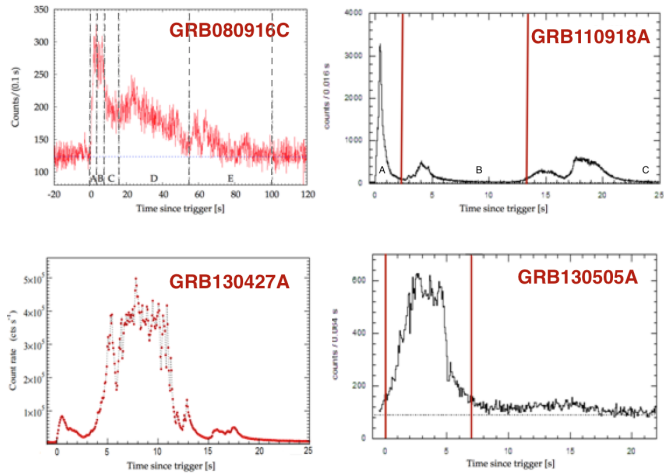

(a)

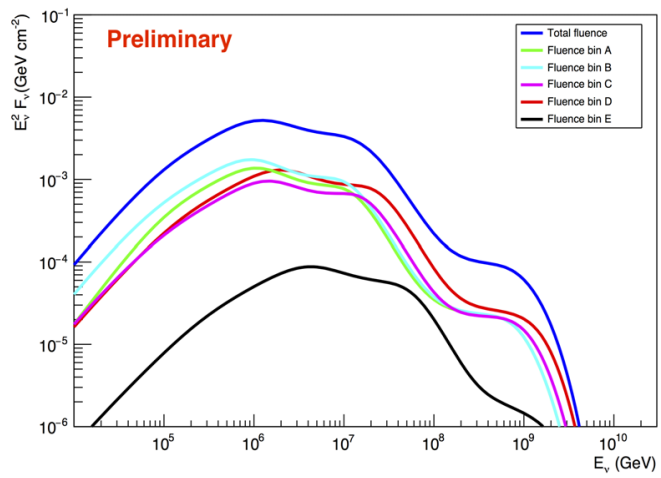

(b)

Figure 2: (a) $\gamma$-ray lightcurves of the selected GRBs. Top left: GRB080916C (Fermi:GBM/LAT [3]); Top right: GRB 110918A (Konus-Wind [4]); Bottom left: GRB 130427A (Konus-Wind [5]); Bottom right: GRB 130505A (Konus-Wind [6]). (b) Time-dependent neutrino spectra of GRB 080916C: $v_{\mu}+\bar{v}_{\mu}$ fluxes are obtained according to NeuCosmA, for the five time bins reported in Tab. 1 .

Table 1: Name of the burst and name of the detector reporting the $\gamma$-ray parameters used in the time-dependent analysis: time bin of the analysis, duration T, fluence $F_{\gamma}$ (in the energy range from $20 \mathrm{keV}$ to $2 \mathrm{MeV}$ for GRB $080916 \mathrm{C}$ and from $20 \mathrm{keV}$ to $10 \mathrm{MeV}$ for the others), low-energy spectral index $\alpha$, high-energy spectral index $\beta$, break energy of the spectrum, redshift $\mathrm{z}$ and variability time scale $\mathrm{t}_{\mathrm{var}}\left({ }^{*}\right.$ is a default value).

\begin{tabular}{cccccccccc}
\hline NAME & Detector & BIN & $\begin{array}{c}\mathrm{T} \\
(\mathrm{s})\end{array}$ & $\begin{array}{c}F_{\gamma} \\
\left(10^{-4} \mathrm{erg} / \mathrm{cm}^{2}\right)\end{array}$ & $\alpha$ & $\beta$ & $\begin{array}{c}E_{\gamma} \\
(\mathrm{keV})\end{array}$ & $\begin{array}{c}\mathrm{z} \\
\text { var } \\
(\mathrm{s})\end{array}$ \\
\hline GRB 080916C & Fermi:GBM/LAT & $\mathrm{A}$ & 3.6 & 0.15 & -0.58 & -2.63 & 440 & 4.35 & 0.23 \\
$"$ & $"$ & $\mathrm{~B}$ & 4.1 & 0.21 & -1.02 & -2.21 & 1170 & $"$ & $"$ \\
$"$ & $"$ & $\mathrm{C}$ & 8.2 & 0.16 & -1.02 & -2.16 & 490 & $"$ & $"$ \\
$"$ & $"$ & $\mathrm{D}$ & 38.9 & 0.53 & -0.92 & -2.22 & 400 & $"$ & $"$ \\
$"$ & $"$ & $\mathrm{E}$ & 46.1 & 0.11 & -1.05 & -2.16 & 230 & $"$ & $"$ \\
GRB 110918A & Konus-Wind & $\mathrm{A}$ & 2.3 & 4.03 & -1.95 & -2.41 & 990 & 0.98 & 0.25 \\
$"$ & $"$ & $\mathrm{~B}$ & 11.0 & 2.06 & -1.00 & -2.60 & 250 & $"$ & $"$ \\
$"$ & & $\mathrm{C}$ & 15.1 & 1.57 & -1.20 & -3.30 & 78 & $"$ & $"$ \\
GRB 130427A & Konus-Wind & - & 18.7 & 26.8 & -0.96 & -4.14 & 1028 & 0.34 & 0.04 \\
GRB 130505A & Konus-Wind & - & 7.0 & 3.13 & -0.69 & -2.03 & 631 & 2.27 & $0.01^{*}$ \\
\hline
\end{tabular}

\section{Analysis methods}

Monte Carlo signal simulations have been performed for each burst, to account for the detector condition at the instrument trigger time. The number of background events $\mu_{b}$ at each GRB position in the angular and temporal search windows was evaluated from data, following the procedure outlined in [8]: the search time window is selected as the whole burst duration plus a symmetric extension of 2 seconds, while the angular window is a cone around the source with semi-aperture $\alpha=10^{\circ}$. In this way the normalized signal and background angular probability density function, $S(\alpha)$ and $B(\alpha)$, are obtained and pseudo-experiments relying on them are performed in both the background only and sig- 
nal plus background hypothesis. At each pseudo-experiment with $\mathrm{n}_{\text {tot }}$ events, an extended maximum likelihood ratio test statistic, defined as [9]

$$
Q=\max _{\mu_{s}^{\prime} \in\left[0 ; n_{\mathrm{tot}}\right]}\left(\sum_{i=1}^{n_{\text {tot }}} \log \frac{\mu_{s}^{\prime} S\left(\alpha_{i}\right)+\mu_{b} B\left(\alpha_{i}\right)}{\mu_{b} B\left(\alpha_{i}\right)}-\mu_{s}^{\prime}\right)
$$

is evaluated in order to obtain the estimated number of signal events $\mu_{s}^{\prime}$. This technique is applied to event samples with different reconstructed track-quality parameter: such a cut is then optimised in order to yield the maximum probability of discovery assuming the NeuCosmA model.

\section{Results and Conclusions}

The expected signal and background event rates for each GRB at the optimal cuts and in the defined search windows are given in Tab. 2: for GRB 110918A, GRB 130427A and GRB 130505A, the expected background is negligible with respect to the predicted signal. After the data unblinding, no neutrino event was observed in temporal and spatial coincidence with any of the GRBs investigated, therefore $90 \%$ C.L. upper limits have been computed on the foreseen neutrino fluence. Defining the differential neutrino fluence $\phi_{v}$, ANTARES limits, expressed as $E^{2} \phi_{v}$, are in the interval $\left[10^{-1}-10\right]$ $\mathrm{GeV} \mathrm{cm}^{-2}$ (Fig. 3). The acceleration of UHECRs in GRBs cannot yet be ruled out. However, some parameters of the model tested here can be constrained [10].

Table 2: Expected number of signal $\mu_{\mathrm{s}}$ and background $\mu_{\mathrm{b}}$ events from each GRB at the optimal cuts.

\begin{tabular}{ccc}
\hline & $\mu_{\mathrm{s}}$ & $\mu_{\mathrm{b}}$ \\
\hline GRB 080916C & $1.8 \times 10^{-3}$ & $8.6 \times 10^{-3}$ \\
GRB 110918A & $1.3 \times 10^{-2}$ & $7.2 \times 10^{-3}$ \\
GRB 130427A & $7.5 \times 10^{-3}$ & $4.1 \times 10^{-3}$ \\
GRB 130505A & $1.6 \times 10^{-1}$ & $2.4 \times 10^{-3}$ \\
\hline
\end{tabular}

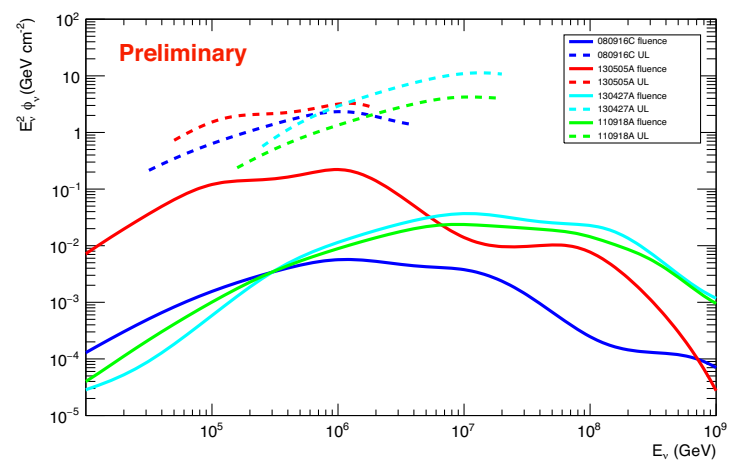

Figure 3: Expected neutrino fluences (solid lines) and ANTARES 90\% C.L. upper limits (dashed lines).

\section{References}

[1] Guetta D et al., Astropart. Phys. 20, 429-455 (2004)

[2] Ageron M at al., Nucl. Instrum. Meth. A 656, 11-38 (2011)

[3] Abdo A et al., Science Mag. 323, 1688 (2009)

[4] Frederiks D et al., Astrophys. J. 779, 151 (2013)

[5] http://gcn.gsfc.nasa.gov/gen3/14487.gen3

[6] http://gcn.gsfc.nasa.gov/gen3/14575.gcn3

[7] Hümmer S, Rüger M, Spanier F and Winter W, Astrophys. J. 721, 630 (2010)

[8] Adrián-Martínez S et al., Astron. Astrophys. 559, A9 (2013)

[9] Barlow R, Nucl. Instr. Meth. A 297, 496 (1990)

[10] Sanguineti M et al., Proceeding of RICAP16, EPJ Web Conf 